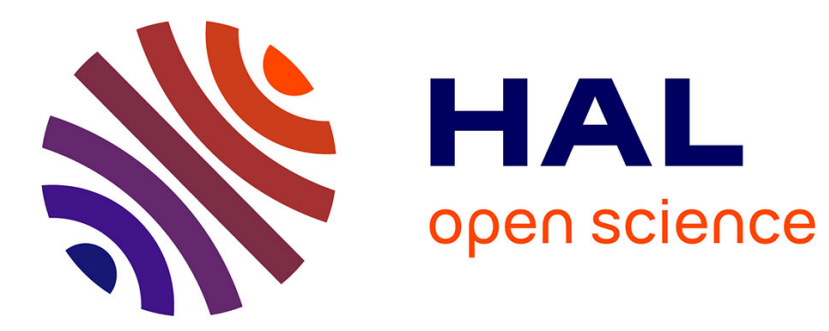

\title{
Quand la répétition se fait figure
}

Véronique Magri-Mourgues, Alain Rabatel

\section{To cite this version:}

Véronique Magri-Mourgues, Alain Rabatel. Quand la répétition se fait figure. Semen - Revue de sémio-linguistique des textes et discours, 2014, 38, pp.7-13. hal-01226603

\section{HAL Id: hal-01226603 \\ https://hal.science/hal-01226603}

Submitted on 9 Nov 2015

HAL is a multi-disciplinary open access archive for the deposit and dissemination of scientific research documents, whether they are published or not. The documents may come from teaching and research institutions in France or abroad, or from public or private research centers.
L'archive ouverte pluridisciplinaire HAL, est destinée au dépôt et à la diffusion de documents scientifiques de niveau recherche, publiés ou non, émanant des établissements d'enseignement et de recherche français ou étrangers, des laboratoires publics ou privés. 
Considérée comme une faute dans l'histoire de la rhétorique ${ }^{1}$ et dans le discours des grammairiens (Frédéric 1985), la répétition est décriée comme pléonasme ou tautologie quand elle associe un caractérisant purement redondant par rapport au caractérisé et dévalorisée quand elle est vue comme source d'un effet de sens négatif, la redondance. L'approche linguistique des répétitions et reprises a depuis longtemps fait litière de ces visions partielles et réductrices (Anderson, Chauvin-Vileno et Madini 2000, Migeot \& Viprey 2000, Le Bot, Schuwer et Richard 2008, Schuwer, Le Bot et Richard 2008). L'approche énonciative des répétitions s'inscrit dans ce mouvement. Dans la continuité des travaux de Bonhomme 2005 et Rabatel 2008b, l'approche énonciative et pragmatique des figures propose une analyse de la répétition qui s'éloigne de la conception référentialiste des mots et du discours et refuse de réduire les figures aux seuls tropes, considérant que la problématique des figures s'intègre dans un processus par lequel les locuteurs ou les énonciateurs ajustent leur rapport cognitif et intersubjectif aux mots, à la réalité et aux allocutaires. Telles sont quelques unes des caractéristiques du cadre théorique énonciativopragmatique qui, dans ce numéro, sert d'arrière-plan à une mise en ordre du fonctionnement syntaxique et sémantique des formes de la répétition. C'est ce projet qui incité les signataires de ce texte à organiser deux journées d'étude autour de la pragmatique des répétitions et de l'analyse de leur fonctionnement dans les genres ${ }^{2}$. Les échanges donnent lieu à cette première publication, centrée d'une part autour des approches textométriques de la répétition et des interprétations herméneutiques auxquelles elles peuvent servir de base, d'autre part autour des approches discursives, conversationnelles et interactionnelles permettant de dégager des effets pragmatiques. Une autre publication suivra qui s'attachera aux répétitions dans les genres, en particulier dans des textes d'ampleur ou de vastes corpus, confirmant l'hypothèse que ces derniers renouvellent l'approche de la réflexion ${ }^{3}$.

Dans la constellation des mots préfixés en re-, reprise, réitération, réduplication, récurrence, reformulation, par exemple, seule une approche contrastive peut contribuer à délimiter le sens différentiel du mot répétition, tout en posant quelques critères définitionnels à même d'esquisser les contours d'une notion.

La portée de la répétition permet de distinguer récurrence et répétition. La récurrence, terme favori de la statistique textuelle, s'appuie sur la notion de fréquence d'une forme, qui mesure la répétition de celle-ci dans le texte, quelle que soit sa position. La portée est indéterminée ou, à tout le moins, n'a aucune incidence sur cette évaluation. Les facteurs positionnels ne sont pas pris en compte dans le calcul de ce que les statisticiens nomment la richesse lexicale (E. Brunet) qui porte en particulier sur les mots pleins que sont les substantifs et qui augmente de manière inversement proportionnelle à la répétition, encore sentie comme indice d'un lexique pauvre et redondant. Au niveau du texte, la lexicométrie permet de déceler un leitmotiv comme retour de

Vaugelas, 1647, Remarques sur la langue française.

2 Ces deux journées ont eu lieu à l'Université Nice Sophia Antipolis, à l'initiative de V. Magri (BCL, UMR 7320) e

d'A. Rabatel (ICAR, UMR 5191) les 5 et 6 décembre 2013.

3 V. Magri \& A. Rabatel (éds), Le Discours et La Langue, 2015, à paraître. 
possibles thèmes concrétisés par les réseaux lexicaux, comme chez Zola. Le calcul des cooccurrences réflexives, autrement dit, les répétitions contiguës d'un terme avec lui-même, impose en revanche que soit délimitée une fenêtre de recherche, équivalant à un fragment de l'espace du texte dont l'empan reste à l'initiative du chercheur. Les linguistes s'accordent pour définir une portée réduite et plus circonscrite à la répétition; la reconnaissance de l'identité de la forme passe par la proximité, dans l'espace du texte, des occurrences répétitives. Cependant, cette condition doit être modulée en fonction des propriétés de l'unité linguistique répétée. La rareté lexicale comme la longueur de cette unité autorisent un éloignement plus grand de l'occurrence $X$ de sa reprise $X^{+1}$, qui n'entravent pas la perception de la répétition. Le sentiment de répétition accompagne l'avancée $\mathrm{du}$ sens sous l'effet de la reconnaissance. De fait, selon l'empan choisi, intraphrastique, interphrastique ou de plus longue portée, la répétition est un facteur de structuration textuelle, que ce soit au niveau microstructural ou macrostructural (A. Mezzadri). Elle joue un rôle architectural et cohésif en établissant une hiérarchisation entre une occurrence-source et les suivantes. Chaque terme présupposant celui qui le précède, la répétition est le moteur de la dynamique textuelle (E. Richard), engagée qu'elle est dans un double mouvement, de retour en arrière vers un déjà-dit d'une part, de nouvelle impulsion qui lance un à-dire d'autre part, tandis qu'à la lecture linéaire se superpose une lecture tabulaire du texte fondée sur une logique sérielle.

Ici quelque chose revient sur soi, quelque chose s'enroule sur soi, et pourtant ne se ferme pas, mais en même temps se libère par son enroulement même. (M. Heidegger, Le Principe de raison, Gallimard, 1962, p. 64)

L'intentionnalité est un autre critère apte à écarter facilement la répétition qui confine à la redite fautive et à la redondance dans un sens non technique ${ }^{4}$. Ce critère évince également la réduplication de mots grammaticaux, envisagée comme la manifestation de ratages du dire dans le discours oral spontané (M.-A. Watine). C'est ce critère qui paraît essentiel pour la détermination du seuil minimal de la répétition. Ce seuil est discutable selon les genres dans lesquels la répétition se trouve réalisée d'une part, selon les propriétés de la répétition d'autre part. En poésie par exemple, genre où le poète ne peut être soupçonné d'aucune négligence ou inadvertance, la répétition fera sens dès $\mathrm{X}^{+1}$; dans d'autres cas, on pourra craindre une reprise de type cognitif par reprise d'un mot présent dans la mémoire courte, sans intention signifiante et c'est seulement à partir de l'occurrence $\mathrm{X}^{+2}$ que la répétition pourra alors être dite figurale. La répétition d'un segment long ou sa place à un poste-clé de la phrase peuvent suffire à justifier sa pertinence, dès $\mathrm{X}^{+1}$. Cela étant, il va de soi que c'est la prise en compte globale des répétitions qui fait sens : l'ensemble des signifiants répétés est pertinent et interprétable.

La saillance enfin érige la répétition en figure du discours, en la dotant d'une plus-value stylistique dont le lecteur doit apprécier le rendement en contexte. Le caractère non aléatoire et volontaire de la répétition lui confère une pertinence, accentuée par des facteurs positionnels quelquefois qui justifient le commentaire. La rhétorique a ainsi clairement assigné des dénominations aux répétitions selon leurs places dans l'énoncé manifestant que la coémergence syntaxique est interprétable en termes figuraux (M. Bonhomme 2005) : anaphore, épiphore, antépiphore, symploque, anadiplose et concaténation font partie par exemple des figures dûment répertoriées. À l'échelle du texte, la répétition peut être envisagée comme matrice d'un discours de campagne (V. Magri) ou comme figure félonienne d'un discours qui se construit comme une boucle déroulant son premier principe et cherchant à y revenir comme à un centre attracteur (A. Mezzadri). Elle peut être aussi un marqueur stylistique, en particulier de l'oralité et de la vocalité dans des récits historiquement situés (M.-A. Watine).

Les domaines d'application différents pour tel ou tel terme établissent, dans certains cas, une distribution d'emploi des termes en re- qui brouillent les essais définitionnels et sont à l'origine de chevauchements terminologiques. Donnée comme synonyme de répétition par le Trésor de la

La redondance grammaticale assure la cohérence du discours. 
Langue française, la reprise, par exemple, est un terme davantage employé en analyse de discours et en analyse conversationnelle et peu enclin à une dimension figurale. Le mot itération, quant à lui, s'avère fort proche de la répétition figurale si on l'envisage comme le résultat d'un processus d'encodage et de décodage (Rastier 1987 : 93) ; sa variante en re-, réitération, peut, elle, s'ouvrir à des réalités autres que linguistique, quand on parle par exemple de la réitération d'une situation.

Le même et la variation. Même si la définition de la reformulation (Gülich 1983, Roulet 1987, Rossari, 1990, 2000, Kara, 2004, Rabatel 2008a) qui pose deux signifiants pour le même signifié paraît établir une différence avec la répétition qui associe a priori le même signifié aux signifiants répétés, les frontières entre les deux procédés sont fragiles, puisqu'ils reposent tous deux sur le binôme identité-variation ou altérité. La reformulation articule un avant-texte auquel elle renvoie anaphoriquement et une expression qui peut être identique formellement mais qui fonctionne comme expression nouvelle pointée comme aboutissement d'un processus discursif. Les répétitions jouent sur la reprise du même - on rejoint le cas de la réduplication stricto sensu, définie comme « répétition littérale, immédiate et iso-fonctionnelle d'un quelconque segment textuel » (Watine 2012: 151) - mais l'inscription dans la chaîne linéaire du texte fait que la seconde occurrence est forcément différente de la précédente : le signifié peut subir une altération de sens en relation avec le cotexte. La deuxième occurrence manifeste un réajustement du dire qui peut se réaliser par exemple par un éventail de prédicats différents qui viennent s'ajouter au même substantif repris dans une visée spécifiante (M.-A. Watine) et un mouvement qui s'assimile à une glose (Julia 2001, Steuckhardt et Niklas Salminen 2003, 2005). La répétition, ductile, joue sur la variation à la manière du motif musical.

\begin{abstract}
Et surtout parce qu'elle [la répétition] est, au sens strict, impossible, un paradoxe : qu'on le veuille ou non, la variation, le changement surgissent de toute répétition même la plus innocente, ne serait-ce que parce que vous changez vous-même en écoutant, ou lisant, ou écrivant ce qui change. C'est cela qui crée la fascination que la répétition exerce, la passion qu'elle peut susciter et l'ennui profond qui guette ceux qui s'y adonnent, double inséparable de la fascination. J. Roubaud, http://licorne.edel.univ-poitiers.fr/document.php?id=3346
\end{abstract}

La problématique de la répétition réside dans ce paradoxe d'une reprise à l'identique, en surface, qui la désigne cependant comme fait d'hétérogénéité énonciative modalisant un premier énoncé ou le mettant en perspective. La répétition se définit donc comme une figure énonciative, une figure à ancrage textuel et discursif. Ce fait d'hétérogénéité énonciative se réalise dans des configurations, écrites ou orales, qui mettent en évidence des situations d'autodialogisme ou d'interaction verbale. L'autodialogisme manifeste, le plus simplement, une réorientation du premier dire pour le confirmer, le renforcer ou l'infirmer. L'énonciation est mise en scène par son dédoublement. La deuxième occurrence ne remplace pas la première mais la remodèle sans l'oblitérer. Dans le cas d'interactions verbales, comme celles des débats radiophoniques (E. Richard), une syntaxe collaborative est à l'œuvre, se construisant entre continuité et rupture. Les échanges dialogaux sont mus par des mécanismes de régulation, des tours de parole qui modèlent le jeu séquentiel des interactions, où se lisent les rapports de force, les négociations discursives, que l'on accepte pleinement ou partiellement les mots de l'autre, autrement dit que le discours se construise en co-énonciation, en sous-énonciation ou en sur-énonciation (A. Rabatel 2008a). Les mots répétés assurent la continuité discursive tout en introduisant une rupture énonciative. Dans les discours de campagne, situation monologale en surface, l'hétéro-répétition entre en scène par l'écho, dans les formules répétées, de patterns socio-linguistiques ou schèmes stéréotypés, formalisés par des patrons syntaxiques minimaux qui construisent le discours en co-énonciation (V. Magri).

La répétition a été envisagée jusque là comme résultat ; elle correspond cependant aussi à un acte d'énonciation, associé à une dimension qu'on pourrait qualifier de performative. Par la vertu de la répétition, on veut faire être le monde, inférant que plus la chose est invoquée, plus elle a de chances de se réaliser, sur le modèle de l'incantation. C'est l'exemple du discours politique qui tente 
moins de persuader que de séduire et d'imposer une vision du monde simplifiée et en écho consensuel avec la mémoire collective convoquée (V. Magri) ; c'est aussi celui du jeu vidéo où l'interaction entre le joueur et son avatar, par le biais de l'encouragement, fait espérer la réussite de l'acte que la parole répète, tout cela par la mise en relation discursive de deux espaces (H. Baldauf). Proche de l'ostinato, qui consiste à répéter une formule rythmique, mélodique ou harmonique, dans le domaine musical, la répétition accompagne l'acte mais s'assimile ce faisant à un mode d'action.

Les contributions de ce numéro s'intéressent toutes à l'aspect figural de la répétition lexicale : les enjeux de la textométrie reposent sur ce postulat que la répétition, envisagée comme récurrence autrement dit comme identité de deux formes, indépendamment de leur position dans le texte, est signifiante. E. Brunet adopte ainsi un point de vue englobant qui traite de la question de la récurrence, évaluée à différents niveaux du texte: le niveau microstructural et la relation intraphrastique, le niveau macrostructural de l'œuvre tout entière, en l'occurrence l'œuvre de Giraudoux, puis l'ensemble des écrivains français avant d'étendre la recherche au très grand corpus numérique de Googlebooks. Les variations d'échelle permettent d'appréhender la répétition comme toute récurrence observable par les outils statistiques.

Cette approche textométrique, fondée sur des calculs contrastifs de fréquences, pose un soubassement sur lequel peuvent s'appuyer les analyses à finalité historique ou herméneutique quand c'est l'interprétation d'une écriture ou d'un imaginaire qui est atteinte au travers de l'analyse de la répétition. C'est la découverte d'A. Mezzadri qui, au moyen d'une analyse lexicométrique outillée par le logiciel IraMuTeQ, met en lumière la répétition lexicale comme une figure qui permet d'appréhender l'infini, caractéristique de l'écriture fénelonienne. C'est l'ambition de M.-A. Watine qui, au travers d'un parcours diachronique de grande ampleur, basé sur Frantext, observe l'évolution des formes concrètes de la répétition comme témoin du changement de la valeur figurale de celle-ci. Elle devient après 1920 un marqueur de dialogisme.

L'étude de V. Magri se focalise sur une figure en particulier, l'anaphore rhétorique, pour en étudier le fonctionnement particulier dans un genre de discours hybride, situé entre écrit et oral, le texte politique de campagne. Cette figure se charge alors de particularités contingentes et déterminées par le genre, pour devenir une véritable forme-sens.

Les deux dernières contributions prennent pour champ d'étude des corpus oraux. E. Richard s'intéresse aux débats radiophoniques : le contexte interactionnel permet de définir la répétition comme figure paradoxale de continuité et de rupture. H. Baldauf, au travers des interventions de joueurs à un jeu vidéo, axe ses remarques sur certaines auto-répétitions pour y voir une figure de l'encouragement.

\section{Bibliographie}

Anderson, Patrick, Chauvin-Vileno, Andrée \& MAdini, Mongi (coord) (2000) : Répétition, altération, reformulation, Besançon, Presses universitaires de Franche-Comté.

Bonhomme, Marc (1998) : Les figures clés du discours, Paris, Éditions du Seuil.

Bonhomme, Marc (2005) : Pragmatique des figures du discours, Paris, Champion.

FRÉDÉRIC, Madeleine (1985) : La répétition. Étude linguistique et rhétorique, Tübingen, Max Niemeyer Verlag.

GÜLICH, Elisabeth \& KOTSCHI, Thomas (1983) : «Les marqueurs de reformulation paraphrastique », Cahiers de linguistique française 5, 305-351.

JULIA, Catherine (2001) : Fixer le sens ? La sémantique spontanée des gloses de spécification du sens, Paris, Presses de la Sorbonne Nouvelle.

KARA, Mohamed (2004) : «Reformulations et polyphonie », Pratiques 123-124, $27-54$.

Le Bot, Marie-Claude, Schuwer, Martine \& RICHARD, Elisabeth (dir) (2008) : La reformulation. Marqueurs linguistiques - Stratégies énonciatives, Rennes, PUR.

MAGRI, Véronique (2012) : «Reformulation et textualité », $3^{\mathrm{e}}$ Congrès mondial de linguistique française, vol. 1 , p. 1143-1159, http://dx.doi.org/10.1051/shsconf/20120100024. 
Migeot, François \& ViPREy, Jean-Marie (éds) (2000) : Répétitions, altérations, reformulation, Semen 12.

MOLINIÉ, Georges (1991) : Éléments de stylistique française, Paris, PUF.

RABATEL, Alain (2008a) : «Stratégie discursive de concordance discordante dans les ensembles reprises + reformulations (en contexte didactique) », in Martine Schuwer, Marie-Claude Le Bot \& Elisabeth Richard (dir), Pragmatique de la reformulation, types de discours, interactions didactiques, Rennes, PUR, 187-202.

RABATEL, Alain (éd) (2008b) : Figures et points de vue, Langue française, 160.

RABATEL, Alain (2012) : «Positions, positionnements et postures de l'énonciateur », Travaux neuchâtelois de linguistique 56, 23-42.

RASTIER, F. (1987). Sémantique interprétative, Paris, PUF.

ROSSARI, Corinne (1990) : «Projet pour une typologie des opérations de reformulation » Cahiers de linguistique française 11, 345-359.

ROSSARI, Corinne (2000) : Connecteurs et relations de discours : des liens entre cognition et signification, Nancy, Presses Universitaires de Nancy.

SCHUwer, Martine, LE Bot, Marie-Claude \& Richard, Élisabeth (2008) : Pragmatique de la reformulation, Rennes, PUR.

Steuckardt, Agnès \& Niklas-Salminen, Aïno (éds) (2003) : Le mot et sa glose, Aix-en-Provence, Publications de l'Université de Provence.

Steuckardt, Agnès \& Niklas-Salminen, Aïno (éds) (2005) : Les marqueurs de glose, Aix-en-Provence, Publications de l'Université de Provence.

WAtine, Marie-Albane (2012) : «La réduplication : une interprétation dialogique », in Frédéric Calas, Catherine Fromilhague, Anne-Marie Garagnon, Laurent Susini (dir), Les figures à l'épreuve du discours, Paris, PUPS, 149160.

WATINE, Marie-Albane (2012 [2013]) : «La réduplication et son contexte », Le discours et la langue 4-2, 61-72. 


\section{Etienne Brunet - Université Nice Sophia Antipolis, BCL, UMR 7320}

\section{La répétition dans la phrase. Etude statistique}

Tout l'univers lexicométrique est fondé sur la répétition. La fréquence d'un mot dans un texte, c'est le nombre qui mesure sa répétition. Or la fréquence des mots et des segments a été considérée généralement à l'échelle large du texte et du corpus, à un niveau où la répétition n'est sensible ni à l'oreille ni à la mémoire. Il convient de se situer aussi à l'échelle du micro texte et de la séquence courte qui s'arrête à la fin de la phrase ou du paragraphe et où se limite la portée de la rémanence mémorielle. On se propose de mener une enquête statistique à ce niveau du microtexte, à travers un roman de Giraudoux, puis de son œuvre entière, avant de s'attaquer à l'ensemble des écrivains français et enfin au corpus français de Google Books.

\section{Summary:}

The entire lexicometric universe is based on repetition. Indeed, the frequency of a word in a text is the measure of its repetition. However, the frequency of words and segments has been generally considered at the broad scale of the text and the corpus, at a level where repetition is neither detectable to the ear nor to memory. It is therefore useful to also examine frequency at the level of the microtext, of the short sequences that terminate at the end of sentences and paragraphs and that fall within the range of the perdurance of memory. We propose performing a statistical investigation at the microtext level, first of a novel by Giraudoux and then of his entire body of work, before undertaking an analysis of all French writers and ultimately the entire French corpus within Google Books.

Mots-clés : textométrie, Giraudoux, Googlebooks, lexicométrie, récurrence, répétition

\section{Agathe Mezzadri}

\section{Répétition lexicale et mémoire : une approche lexicométrique de l'infini dans les Fuvres complètes de Fénelon}

Large corpus numérique dont l'aspect répétitif a été souligné par les commentateurs, les CEuvres complètes de Fénelon offrent un terrain propice à une réflexion sur les liens unissant la répétition intra-phrastique de mots simples à la mémoire. L'analyse lexicométrique au moyen du logiciel IRaMuTeQ permet à cet égard une problématisation du phénomène et une série de résultats chiffrés. La modélisation linguistique de la répétition lexicale y vacille en raison de questions de distance entre les différentes itérations, eux-mêmes liés à des problèmes psycholinguistiques de mémoire à court terme. Elle oscille également entre trois définitions issues des domaines de la linguistique, du langage fénelonien et de la tradition rhétorique. L'exploration statistique puis manuelle de ces diverses acceptions permet de mettre en valeur la spécificité stylistique du corpus dans son rapport à l'infini. Peu attaché aux figures traditionnelles de la répétition, une figure du discours nouvelle en émerge, caractérisée par l'entrelacement dense et infini de mêmes mots, s'écoulant de son « premier principe » ou « source » et y retournant inlassablement.

Mots-clés : Répétition intra-phrastique, Mémoire linguistique, Infini, Fénelon, Lexicométrie 
Abstract: The Complete Works of Fenelon offer a fertile ground to reflect on the links between repetition of simple words and memory. Indeed, commentators underlined the repetitive aspect of this corpus. Also, its largeness allows questioning the lector's identification of repetitions. It raises the issue of a minimum distance between the different iterations, this issue being related to psycholinguistic problems of short-term memory. Using the software IRaMuTeQ on this corpus obliged us to question three different approaches of the phenomenon and provided us with a series of results. Thence, a stylistic specificity of the corpus emerges in its report to infinity. It lays on the creation of a new figure of speech. Far from the traditional figures of repetition, this one is characterized by dense and infinite interlacing of the same words flowing from their "first principle" or "source" and returning endlessly to it.

Key words: Repetition, Linguistic memory, Infinity, Fénelon, Lexical statistics

Marie-Albane Watine - Université Nice Sophia Antipolis, BCL, UMR 7320

\section{Les âges de la réduplication}

Les figures de répétition ont une vie propre, elles connaissent un avènement, et subissent des phénomènes de mode ou de désaffection. C'est sur l'historicisation d'une figure et de ses différentes réalisations que cette contribution met l'accent, en se limitant à l'étude de la réduplication, ou répétition immédiate et iso-fontionnelle d'un quelconque segment textuel, dans l'écrit littéraire.

En s'appuyant sur l'ensemble du corpus Frantext, elle montre que cette figure apparaît tardivement, vers le milieu du XIXe siècle, et qu'elle est liée aux expérimentations touchant à la stylisation du parlé. A partir de 1920, elle subit peu de changements quantitatifs, mais elle évolue radicalement d'un point de vue typologique. Alors qu'elle ne touchait que certaines classes grammaticales et pouvait globalement s'interpréter de façon intensive, elle se complexifie d'un point de vue énonciatif et devient un véritable marqueur de dialogisme. Dans les décennies 1920-1960, apparaissent successivement trois nouveaux sous-types de réduplication (reprise-écho hétérodialogique, reprise auto-dialogique, parasitage de l'à-dire) qui exploitent pleinement la capacité de la figure à mettre en présence des termes identiques en surface, mais porteurs de points de vue divers. L'étude tente d'expliquer cette créativité typologique par des déterminations linguistiques, culturelles et esthétiques.

\section{Mots-clés}

Répétition, réduplication, épizeuxe, oralité dans la littérature

Figures of speech based on repetition have a life of their own, they experience a rise, they are subject to trends of fashion and may at times outdate; the pragmatic approach has an interest to take into account this historicity of forms and functions, which determines the overall framework of specific effects. This contribution focuses on the typological evolution, in litterary texts, of one particular figure called reduplication, defined as an immediate repetition of any textual segment with no grammatical function variation.

On the basis of an original corpus of several thousand occurrences constituted from Frantext, the study shows that reduplication spreads in the mid nineteenth century, and can be related to the stylisation of spoken language in littérature. Since 1920, it doesn't evolve quantitatively, but undergo some radical typological changes. Three new subtypes emerge that shows the enunciative complexity of a figure hitherto restricted to an effect of intensification. The study attemps to explain this typological creativity through linguistic and aesthetic determinations.

\section{Keywords}




\section{Véronique Magri - Université Nice Sophia Antipolis, BCL, UMR 7320 \\ L'anaphore rhétorique dans le discours politique - l'exemple de N. Sarkozy}

L'article analyse l'anaphore rhétorique dans le discours politique. Le corpus d'étude est constitué des discours de campagne de N. Sarkozy. Après avoir évalué les critères définitionnels qui font de l'anaphore une figure structurante, les enjeux pragmatiques de cette dernière sont observés. Figure de structuration, d'amplification et d'assertivité, elle peut être définie comme forme-sens signifiante dans un genre particulier. L'approche de l'anaphore rhétorique est renouvelée par cette analyse qui croise pragmatique, syntaxe mais aussi analyse quantitative.

Mots-clés : Répétition, discours politique, anaphore, pragmatique

\section{Rhetoric anaphora in political speech - the example of N. Sarkozy}

The purpose of this paper is to analyse rhetoric anaphora in the political speech. The analysis will be carried out on a corpus composed by the election campaign speeches of N. Sarkozy. First the definitional criteria of the anaphora as a structural figure are tested ; then its pragmatical function is observed. The anaphora is a structural, hyperbolic and assertive figure of speech. It can be defined as a significant pattern in a particular genre. This approach is renewed by this paper which meets pragmatics, syntax but also quantitative analysis.

Key-words : repetition, political speech, rhetoric anaphora, pragmatics

\section{Elisabeth Richard - Université Rennes 2, LIDILE EA 3874}

À propos de répétition : entre continuité et rupture

Considérant la répétition lexicale comme le retour à l'identique, en position seconde, d'un même item lexical, l'article interroge les notions de continuité et de rupture qui lui sont inhérentes. Ainsi, après avoir observé certaines formes de répétition chez un même locuteur, l'article propose d'analyser plus précisément les répétitions qui interviennent dans les interactions des débats radiophoniques. Il met en outre au jour des schémas de répétitions qui rappellent du « déjà-dit » et engagent un à-dire. Lieux de transition, les schémas du type «à propos de $\mathrm{X}_{\text {répété }}$ s sont également des lieux privilégiés pour étudier les postures énonciatives différenciées qui se dégagent dans les échanges radiophoniques.

Mots clés : répétition, syntaxe, débat, transition, argumentation

\section{Repetition : between continuity and rupture}

Lexical repetition is defined as the occurrence of a given lexical item a second time. This study deals with the notions of continuity and rupture that are inherent to repetition. Thus, after observing certain patterns of repetition by the same speaker, we analyze here the repetitions that occur in the interactions characteristic of radio call-in discussions. The study reveals repetition patterns which recall something that has already been said (« déjà-dit») and pave the way for something to be said

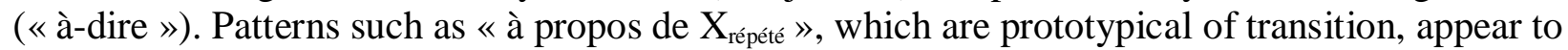
also be valuable objects to study utterers' postures in radio debates.

Key words : repetition - syntax - argumentativ dimension - transition -

Heike Baldauf-Quilliatre, Lyon 
L'article s'intéresse au rôle et au fonctionnement de la répétition dans la construction d'énonciations exprimant un encouragement lors d'un sport collectif. Il s'agit d'un type d'auto-répétitions très particulier : des unités de parole plutôt courtes sont répétées une ou plusieurs fois en contiguïté et sous une même courbe prosodique. Elles forment une seule unité et accomplissent une seule action. L'élément non-répété exprime dans la majorité des cas une instruction ou une évaluation, c'est la répétition associée à des schémas prosodiques et lexicaux, à différents procédés praxéologiques ou multimodaux et à des positions séquentielles spécifiques qui en fait un encouragement. L'article met l'accent sur les aspects qui font de la répétition par excellence pour l'expression d'un encouragement : iconicité et intensité. L'analyse se base sur un corpus d'interaction de joueurs d'un jeu de football sur une console de jeu.

Mots-clés : multiple sayings, encouragement, jeux vidéo, iconicité, intensité

\section{Repetition and encouragement}

The paper examines the role and the functions of repetitions in the construction of utterances, which express an encouragement in a collective sport. It focuses at self-speaker-repetition as multiple sayings: rather short units of talk are repeated once or several times, following each other immediately and being pronounced under one single prosodic contour. Together they form one single turn constructional unit and accomplish one single action. The repeated element expresses at its own mostly an instruction or an assessment. Only the repetition allied to particular prosodic and lexical patterns, praxeological or multimodal procedures and specific sequential positions creates the encouragement. The paper highlights those aspects of repetition, which are most important for the expression of encouragement: iconicity and intensity. The analysis is based on a corpus of video players' interaction during a football game on a game console.

Keywords: multiple sayings, encouragement, video games, iconicity, intensity 